\title{
Modelling and simulation of the rutting resistance of bituminous mixes: experimental and stochastic approaches
}

\author{
A. E. Ouni ${ }^{1,2}$, A. Dony ${ }^{2} \&$ J. Colin ${ }^{2}$ \\ ${ }^{I}$ Arts et Métiers ParisTech (ENSAM), France \\ ${ }^{2}$ Ecole Spéciale des Travaux Publics, du Bâtiment et de l'Industrie \\ (ESTP), Institut de recherche en constructibilité (IRC), France
}

\begin{abstract}
This work deals with the simulation of the rutting resistance of bituminous binders used in road pavement. First, the experimental protocol was assessed to simulate pavement traffic allowing the prediction of the rutting depth evolution versus cycle number of wheel passes under isothermal conditions. Then a probabilistic parametric approach was developed to take into account the different parameter uncertainties related to the changes in experimental conditions. We investigate through the stochastic approach the rutting sensitivity of bituminous mixes under traffic load. A confidence region of a high probability of $99 \%$ is defined to allow the prediction of the in situ rutting potential of bituminous specimens.
\end{abstract}

Keywords: rutting, bitumen, uncertainties, stochastic.

\section{Introduction}

The environment protection has been and continues to be the major concern of the road politics in industrialized countries. With the signature of the Kyoto protocol agreement, the European Union is involved to reduce the energy consumption and the resulting greenhouse gases emissions [1]. The warm mix asphalt (WMA) is a promising technology which has been developed to contribute to the protection of the environment and sustainable development program [2-5]. The WMA is a mixture of mineral aggregates and bitumen produced and placed on the road at a lower temperature comparing to the traditional hot mix asphalt (HMA). Reductions in temperature of $20-50^{\circ} \mathrm{C}$ have 
been recorded. As a result, significant benefits were noted through the energy saving and the reduced emissions from burning fuels, undesirable fumes and odors generated at the plant and paving site. Typically, the HMA is produced at $140^{\circ} \mathrm{C}-180^{\circ} \mathrm{C}$ to make the binder viscous enough to coat the aggregate and to ensure a good workability of the asphalt during laying and compaction. In the case of WMA, there are some techniques developed and used in European countries to produce mix asphalt at low temperature without altering the workability of the mixture [6].

During its service life, the road pavement experiences multiple failure modes such as permanent deformation (rutting), fatigue and thermal cracking [7, 8]. The rutting phenomenon occurring at high service temperature is essentially due to the mechanical traffic load. The accumulated strains resulting from the vehicle passes lead to a plastic deformation in pavement layer. Due to the excessive pavement rutting, water and snow can stagnate in the ruts, for example, and lead to vehicle hydroplaning and accidents. Accordingly, the WMA is required to exhibit a good rutting performance once in pavement. In addition, the laboratory measurements of rutting exhibit very often some dispersion linked to the experimental uncertainties. It was shown by the authors in a previous work [9] that this probabilistic aspect has to be taken into account in order to have an accurate prediction of asphalt rutting performance through bitumen characteristics.

In this context, the aim of this paper is to emphasize though a rutting test carried on warm and hot bituminous mixtures the necessity to consider the experimental uncertainties in order to quantify the random response related to the rut. Suitable probability distributions were developed on the light of the statistical information derived from experiments to describe the random stochastic variable attributed to the rut.

\section{Experimental program to evaluate rutting performance}

\subsection{Material description}

This study is carried out by considering a Semi-coarse asphaltic concrete (BBSG 0/10) formulated as follow:

Table 1: Composition of asphalt mixture.

\begin{tabular}{|c|c|c|c|c|}
\hline $0 / 2$ Noubleau & $2 / 6$ Noubleau & $6 / 10$ Noubleau & $\begin{array}{c}\text { Filler calcaire } \\
\text { Piketty }\end{array}$ & $\begin{array}{c}35 / 50 \\
\text { Bitumen }\end{array}$ \\
\hline $33 \%$ & $20 \%$ & $45 \%$ & $2 \%$ & $5 \%$ \\
\hline
\end{tabular}

The bituminous mixture specimens were prepared in laboratory (ESTP France). First, hot mix asphalt (HMA) was prepared by mixing aggregates together with bitumen at a mixing temperature of $165^{\circ} \mathrm{C}$. Then warm mix asphalt (WMA) was obtained by mixing the components at $110^{\circ} \mathrm{C}$. During the preparation of WMA and in order to improve the workability of the asphalt 
binder so that it can be sufficiently viscous to coat the aggregate, a surface active additive was incorporated at $0.3 \%$ into the bitumen.

\subsection{Laboratory compaction}

To produce representative specimens for rutting test, slabs were manufactured with the prepared bituminous mixes and afterwards compacted in moulds by a roller compactor device (LCPC - France) according to the standard specifications (NF EN 12679-33). The apparatus (Fig. 1) provides a pneumatically powered means of compacting slabs to reproduce in situ compaction. The dimensions of the obtained slabs are $500 \mathrm{~mm}$ x $180 \mathrm{~mm}$ x $100 \mathrm{~mm}$.

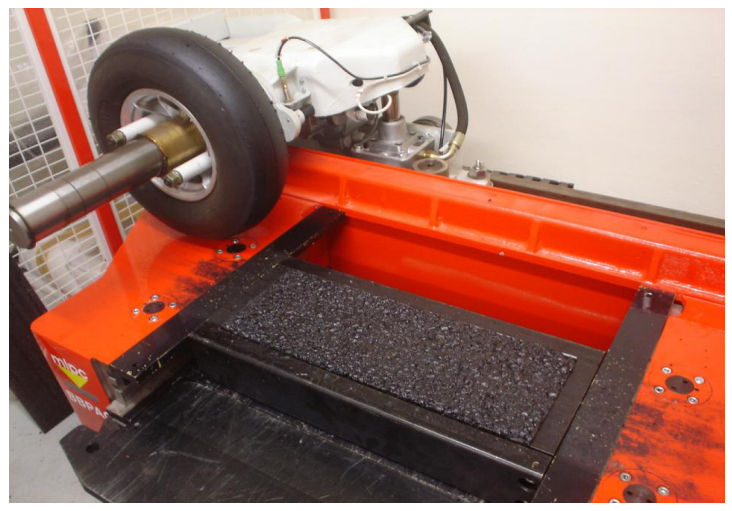

Figure 1: $\quad$ Slabs roller compactor.

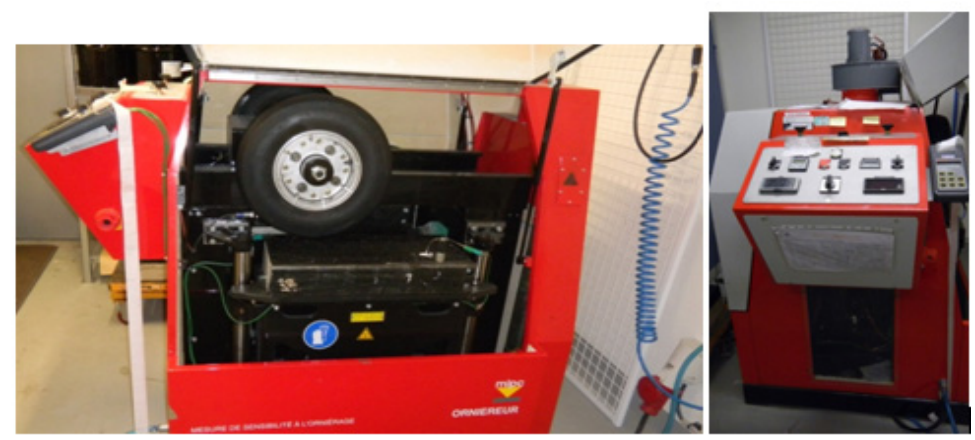

Figure 2: $\quad$ French rutting test device.

\subsection{Rutting test}

There are several types of wheel tracking devices that can be used to evaluate the rutting potential of a mixture. The three most known laboratory devices are the Asphalt Pavement Analyser (APA), the Hamburg Wheel Tracking Device (HWTD) and the French Public Works research Laboratory (LCPC) wheel 
tracker commonly known as the French Rutting Tester (FRT). For further information on these devices, the reader could consult [10]. Here, the FRT is used to evaluate the permanent deformation of the bituminous mixtures. This device has been used in France for over 15 years to simulate the traffic load through repetitive passes of a wheel pressurized to $0.6 \pm 0.03 \mathrm{MPa}$. The FRT (Fig. 2) tracks across an asphalt specimen (slab) a loaded wheel of $500 \mathrm{~N}$ for many thousands of cycles. The rutting test was performed conforming to the standard specifications (NF EN 12679-22).

Accordingly, the temperature was monitored uniformly and maintained to $60^{\circ} \mathrm{C}$ by temperature sensors inserted within the slab while local deformation is continuously recorded by depth gauge. Here, 2 slabs of HMA and WMA mixtures were tested simultaneously on the rutting device. After the required number of cycles, the rut depth was measured in $\mathrm{mm}$ at 15 different positions on the slab and then an average value of the rut is considered. Here, a load cycle corresponds to an outward and return motion of the wheel. In the following, ri denotes the local rut depth measured at the position (i) on the slab. The rut after $n$ cycles is expressed in percent (\%) according to eqn (1):

$$
\text { Rut }_{\mathrm{n}}(\%)=\sum_{\mathrm{i}=1}^{15} \frac{(\mathrm{ri}-\mathrm{r} 0 \mathrm{i})}{15 \mathrm{xh}} \times 100
$$

where r0i represents the initial measurements at the positions (i) located on the slab and $\mathrm{h}$ its thickness.

In figure 3, we present the evolution of the rut depth in percent versus the number of cycles for both hot and warm asphalt mixes. One can see that the 2 mixtures exhibit nearly the similar performance with a better rutting resistance of the HMA particularly with the increasing of the cycles number. In fact, we recorded after 30000 cycles a rut depth of $4.23 \%$ for the HMA versus $6.53 \%$ in the WMA case. We note that in the French specifications [8], the maximum of rut depth shall be less than $10 \%$. This constraint is marked by a dark continuous line in figure 3 .

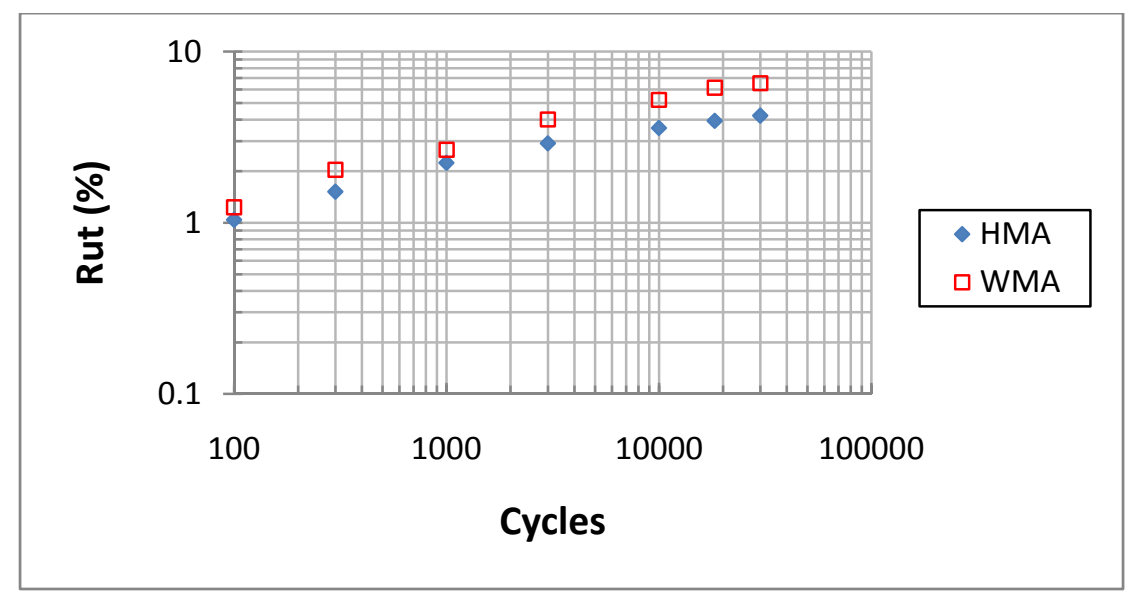

Figure 3: Rutting test results. 


\section{Parametric probabilistic modelling of experimental uncertainties}

\subsection{Stochastic approach}

In this section, we will make use of the experimental uncertainties derived from the aforementioned rut depth values measured on the top surface of the slab. The 15 local measurements ri recorded after $n$ cycles allow the definition of average and standard deviation values for the rut depth. In the following, we will denote by $\mathbf{R}$ (in bold letters) the random variable associated to the rut in percent. In order to describe the random uncertainties of this variable, the entropy maximum principle (EMP) is considered for the probabilistic parametric modeling $[11,12]$. In general, given a random variable $\mathrm{X}$, The EMP allows the construction of adequate and realistic probability density functions ( $\mathrm{pdf}$ for short) of $\mathrm{X}$ on the light of the given information. Accordingly, the dispersion of $\mathrm{X}$ is described by the pdf $\mathrm{p}_{\mathrm{X}}$ and it is quantified by the entropy $\mathrm{S}$ :

$$
S(X)=-\int_{-\infty}^{+\infty} p_{X}(x) \log \left(p_{X}(x)\right) d x
$$

The given or available information may be the average (or mean) of the variable, the standard deviation, the second or higher order moments, etc. The entropy maximum principle states that the suitable probability density function which describes the random variable distribution is obtained by maximizing the entropy S. This leads to an optimization problem constrained by the known information formulated as follow:

$$
\left\{\begin{array}{l}
\text { Maximize } \mathrm{S}(\mathrm{X}), \text { subject to: } \\
\int_{-\infty}^{+\infty} p_{X}(x) d x=1 \\
f_{i}=\int_{-\infty}^{+\infty} g_{i}(x) p_{X}(x) d x \quad \mathrm{i}=1 \text { to } \mathrm{n},
\end{array}\right.
$$

where $f_{i}$, represent the available information. For instance, if $f_{i}=m_{X}$ (mean of the random variable $X), g(x)=x$ whereas when $f_{i}=m_{2}$ (second order moment), $g(x)$ $=\mathrm{x}^{2}$.

This optimization problem can be solved by minimizing a Lagrangian function $H$ which includes $(1+n)$ Lagrange multipliers $\left(\lambda_{0}, \lambda_{i}, i=1\right.$ to $\left.n\right)$ associated to the $(1+n)$ constraints. The function $H$ is given by:

$H\left(\lambda_{0}, \lambda_{1}, \ldots, \lambda_{n}\right)=\lambda_{0}+\sum_{i=1}^{n} f_{i}+\int_{-\infty}^{+\infty} \Pi_{[a, b]}(x) \exp \left(-\lambda_{0}-\sum_{i=1}^{n} \lambda_{i} g_{i}(x)\right)$

where $[a, b]$ denotes the support of the random variable and $\Pi_{[a, b]}$ an indicator function defined as: 


$$
\Pi_{[a, b]}(\mathrm{x})=\left\{\begin{array}{c}
1 \text { if } \mathrm{x} \in[\mathrm{a}, \mathrm{b}] \\
0 \text { if not }
\end{array}\right.
$$

Once $\lambda_{i}$ are found, the probability distribution of the random variable is known and it is given by:

$$
p_{X}(x)=\Pi_{[a, b)}(x) \exp \left(-\lambda_{0}-\sum_{i=1}^{n} \lambda_{i} g_{i}(x)\right)
$$

\subsection{Results}

To illustrate this methodology, we focus on the rutting results obtained at 30000 cycles. The average and standard deviation values of the rut for Hot and warm bituminous mixtures are recapitulated in table 2 .

Table 2: $\quad$ Statistical data of the rut in percent.

\begin{tabular}{|c|c|c|}
\cline { 2 - 3 } \multicolumn{1}{c|}{} & Average_rut & Standard deviation_rut \\
\hline HMA & 4.23 & 1.62 \\
\hline WMA & 6.53 & 0.74 \\
\hline
\end{tabular}

In our case, given the average and the STD values and assuming that the random variable associated to the rut depth shall not take nor negative neither infinite value, the minimization of Eq. (x) leads to a Gaussian distribution:

$$
p_{R u t}(r)=\exp \left(-\lambda_{0}-\lambda_{1} r-\lambda_{2} r^{2}\right)
$$

Figure 4 presents the probabilistic distributions as well as numerous random trials (1000 in total) of the rut according to the probability density function for both hot and warm asphalt mixes. The red continuous line corresponds to the average value of the rut in percent. Analogically, we can follow the same methodology to determine the rut pdf after 100, 300, 1000...cycles. Then, after a given number of cycles, we carry out some random realizations according to the pdf. This will be useful to define a confidence region including the probabilistic responses of the rut.

In figure 5, we show the random variation of the rut value versus the number of cycles for HMA and WMA cases. As can be seen, the mean (or deterministic) model using the average values of the rut lies in the middle of the confidence region. In addition, one can note that even though the confidence regions of the 2 mixtures are very close at the beginning of the test which seems to be positive for warm asphalt rutting performance, the discrepancy increases for higher number of cycles where the intervals become more spaced. 

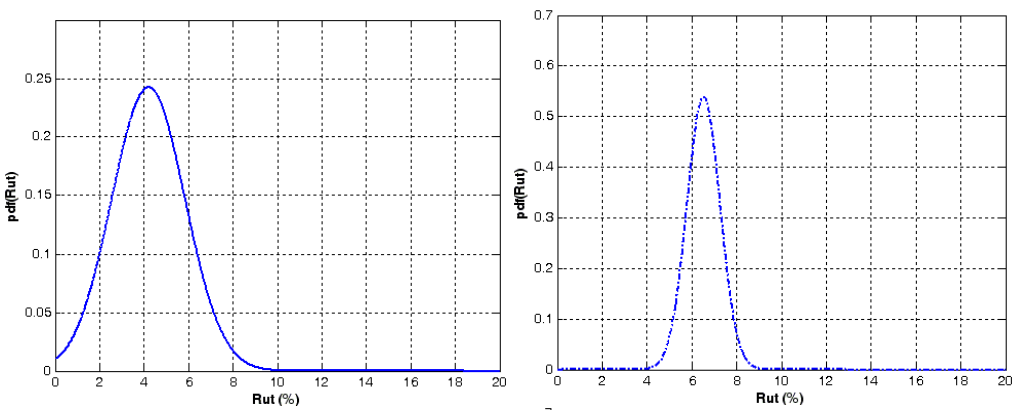

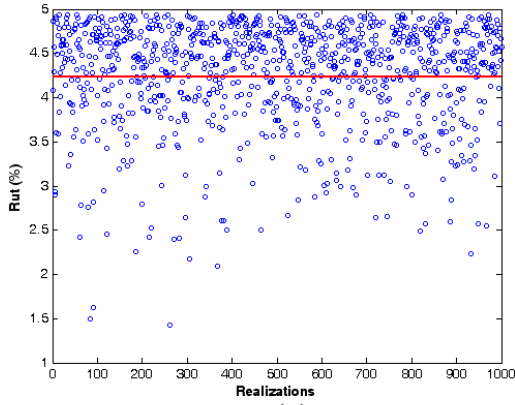

(a)

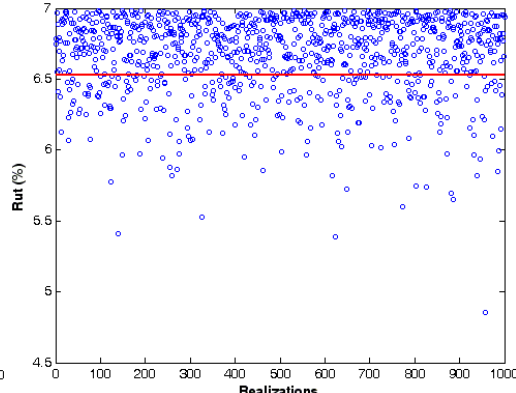

(b)

Figure 4: Probabilistic density functions of the rut and random realizations, (a) HMA, (b) WMA.

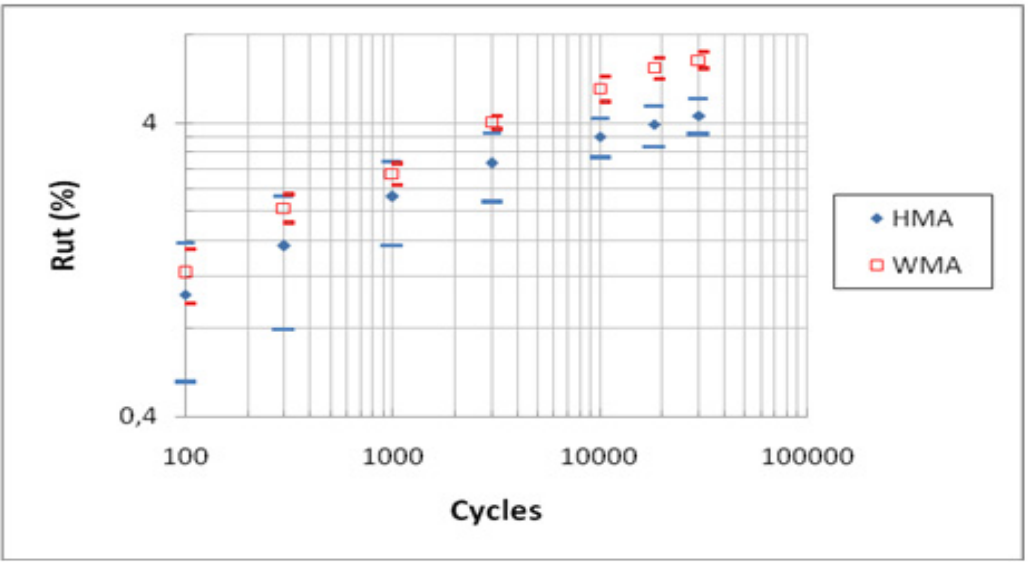

Figure 5: Confidence regions for rut.

\section{Conclusion}

A series of experimental tests were performed in this paper to compare the rutting performance of HMA and WMA obtained by the incorporation of surface 
active additive. The rutting test FRT shows that both asphalt mixes exhibit comparable rutting performance after 30000 loaded cycles. This finding added to the environmental advantageous outlines the beneficial use of warm mix asphalt technology in road pavement. The stochastic approach was developed on the light of the available statistical information derived from the experimental uncertainties linked to the rut measurements. Accordingly, confidence regions were defined to estimate the probabilistic rut depth response with respect to the adequate probability density function. Discrepancies in confidence intervals are noticed between the 2 mixtures especially for a high number of wheel passes.

In the future, it would be interesting to investigate the modification of rheological and chemical properties of the bituminous binders recovered from the mixtures. This study could improve our knowledge and understanding of the bitumen ageing phenomenon.

\section{References}

[1] International Technology Scanning Program, Warm-Mix Asphalt: European Practice, 2008.

[2] Brosseaud Y., Ecologiques, sécuritaires, confortables, les enrobés de demain se feront autrement : Présentation des enrobes tièdes, 2006.

[3] Collectif. Un sujet chaud, les enrobés basses calories. Bitume info. $\mathrm{N}^{\circ} 12$, pp. 12-15, 2006.

[4] Brosseaud Y., Warm asphalt-Overview in France. LCPC, France, Présentation to WMA scan team, 2007.

[5] Brosseaud Y. \& Saint Jacques M, Warm Asphalt Mixes: Overview of This New Technology in France, Second European Road. Transport Research Arena Europe, Ljubljana, Slovénie, 21-24, 2008.

[6] Harder, G. et al (2008). Energy and environmental gains of warm and halfwarm asphalt mix: quantitative approach. In: Transportation Research Board, Washington D.C., 2008.

[7] "Warm mix asphalt technologies and research", Government engineering, July-August, 2007.

[8] Perraton D., Di Benedetto H., Sauzéat C. et al., Rutting of bituminous mixtures: wheel tracking tests campaign analysis. Materials and structures, Online First, 2010.

[9] Eddhahak Ouni A., Dony A. \& Colin J., Assessment of a probabilistic parametric rheological model to predict the rutting resistance of bitumen, ASMDO 2010.

[10] Cooley Jr., Allen L., Prithvi S. Kandhal, \& M. Shane Buchanan. Loaded Wheel testers in the United States: State of the Practice. Transportation Research Board E-Circular number E-C016, 2000.

[11] Shannon, C.E., A Mathematical Theory of Communication, Bell System Technical Journal 27, pp. 379-423 \& 623-656, 1948.

[12] Jaynes, E.T., Information theory and statistical mechanics, Physical Review 106, pp. 620-630, 1957. 